\title{
Pharmacotherapy of atrial fibrillation in COVID-19 patients
}

\author{
Anna Tomaszuk-Kazberuk ${ }^{1,2}$, Marek Koziński, ${ }^{1,3}$, Justyna Domienik-Karłowicz ${ }^{1,4}$, \\ Miłosz Jaguszewski ${ }^{1,5}$, Szymon Darocha ${ }^{1,6}$, Maciej Wybraniec ${ }^{1,7}$, \\ Piotr Dobrowolski ${ }^{1,8}$, Karolina Kupczyńska ${ }^{1,9}$, Błażej Michalski ${ }^{1,9}$, \\ Wojciech Wańha ${ }^{1,10}$, Agnieszka Kapłon-Cieślicka ${ }^{11}$
}

\begin{abstract}
1“Club 30", Polish Cardiac Society, Poland; ${ }^{2}$ Department of Cardiology, Medical University of Bialystok, Poland; ${ }^{3}$ Department of Cardiology and Internal Medicine, Medical University of Gdansk, Gdynia, Poland;

${ }^{4}$ Department of Internal Medicine and Cardiology with the Center for Diagnosis and Treatment of Thromboembolism, Medical University of Warsaw, Poland; ${ }^{5} 1^{\text {st }}$ Department of Cardiology, Medical University of Gdansk, Poland; ${ }^{6}$ Department of Internal Medicine and Cardiology, Medical University of Warsaw, Poland; ${ }^{7} 1^{\text {st }}$ Chair and Department of Cardiology, School of Medicine in Katowice,

Medical University of Silesia, Katowice, Poland; ${ }^{8}$ Department of Hypertension, National Institute of Cardiology, Warsaw, Poland; ${ }^{9}$ Chair and Department of Cardiology, Medical University of Lodz, Poland; ${ }^{10}$ Department of Cardiology and Structural Heart Diseases, Medical University of Silesia, Katowice, Poland;

${ }^{11} 1^{\text {st }}$ Chair and Department of Cardiology, Medical University of Warsaw, Poland
\end{abstract}

\section{This paper was guest edited by Prof. Janina Stępińska}

\begin{abstract}
The coronavirus pandemic disease 2019 (COVID-19) has changed the face of contemporary medicine. However, each and every medical practitioner must be aware of potential early and late complications of COVID-19, its impact on chronic diseases - especially ones as common as atrial fibrillation $(A F)$ and the possible interactions between patients' chronic medications and pharmacotherapy of COVID-19. Patients with $A F$ due to comorbidities and, often, elderly age are assumed to be at a higher risk of a severe course of COVID-19. This expert consensus summarizes the current knowledge regarding the pharmacotherapy of AF patients in the setting of the COVID-19 pandemic. In general, anticoagulation principles in quarantined or asymptomatic individuals remain unchanged. Nevertheless, it is advisable to switch from vitamin K antagonists to non-vitamin K antagonist oral anticoagulants (NOACs) whenever possible due to their consistent benefits and safety with fixed dosing and no monitoring. Additionally, in AF patients hospitalized due to mild or moderate COVID-19 pneumonia, we recommend continuing NOAC treatment or to switching to low-molecular-weight heparin (LMWH). On the other hand, in severely ill patients hospitalized in intensive care units, intravenous or subcutaneous dosing is preferable to oral, which is why the treatment of choice is either LMWH or unfractionated heparin. Finally, particularly in critical scenarios, the treatment strategy in COVID-19 patients with AF should be individualized based on possible interactions between anticoagulants, antiarrhythmics, antivirals, and antibiotics. In this consensus, we also discuss how to safely perform COVID-19 vaccination in anticoagulated $A F$ patients. (Cardiol J 2021; 28, 5: 758-766)
\end{abstract}

Key words: atrial fibrillation, coronavirus, infection, severe acute respiratory syndrome coronavirus-2 (SARS-CoV-2), coronavirus disease 2019 (COVID-19), non-vitamin K antagonist oral anticoagulants (NOACs), direct oral anticoagulants (DOACs)

Address for correspondence: Justyna Domienik-Karłowicz, MD, PhD, Department of Internal Medicine and Cardiology with the Center for Diagnosis and Treatment of Venous Thromboembolism, Medical University of Warsaw, ul. Lindleya 4, 00-005 Warszawa, Poland, tel: +48 2250211 44, fax: +48 2250213 63, e-mail: jdomienik@tlen.pl

Received: 14.12.2020 Accepted: 30.06.2021 Early publicaion date: 9.08.2021

This article is available in open access under Creative Common Attribution-Non-Commercial-No Derivatives 4.0 International (CC BY-NC-ND 4.0) license, allowing to download articles and share them with others as long as they credit the authors and the publisher, but without permission to change them in any way or use them commercially. 


\section{Introduction}

The coronavirus pandemic disease (COVID-19) has changed the face of contemporary medicine. After the initial turmoil in hospitals and paralysis of the ambulatory healthcare system in Poland, relevant (although not always sufficient) strategies, including telemedicine and eHealth solutions in ambulatory care, and - in non-specialty hospitals - procedures to ensure adequate management of medical emergencies and urgencies have been implemented. Still, access to both outpatient and inpatient care remains markedly restricted and aggravated by fear of infection, further discouraging patients from seeking medical assistance. This situation needs to be remedied as soon as possible to enable appropriate medical care of "chronic" patients, preventing them from developing acute disease exacerbations, long-term complications, or adverse effects from unsupervised treatment. Nevertheless, there is a need to be vigilant about the possibility of COVID-19 infection in patients and acknowledge that this disease will stay with us for the next few months or - more probably - years. Thus, each and every medical practitioner - irrespective of specialty or place of practice (ambulatory care, a specialty hospital, non-specialty hospital) - must be aware of potential early and late complications of COVID-19, its impact on chronic diseases, and possible interactions between patients' chronic medications and pharmacotherapy of COVID-19.

Older age and cardiovascular diseases are known predictors of a severe course of COVID-19 $[1,2]$. Atrial fibrillation (AF) itself has not been identified as an independent risk factor; however, $\mathrm{AF}$ patients are largely elderly individuals burdened with concomitant diseases, including hypertension, diabetes, heart failure, and/or coronary artery disease, which are demonstrably related to higher morbidity and mortality in the course of severe acute respiratory syndrome coronavirus-2 (SARS-CoV-2) infection [1, 2]. These patients more often require hospitalization for COVID-19 and antiviral treatment. Furthermore, up to $10 \%$ of patients with acute respiratory distress syndrome develop new-onset AF [3]. Data in SARS-CoV-2 cases are still scarce, but so far AF was reported in 1 in 5 patients hospitalized for COVID-19, with higher rates in severe cases [4-6]. Thus, a high proportion of patients treated for COVID-19 might be receiving medications for $\mathrm{AF}$, which carries a high potential for drug interactions.
This expert consensus aims to summarize the current knowledge regarding the pharmacotherapy of AF patients in the setting of the COVID-19 pandemic. Of particular focus herein, on optimal anticoagulation in $\mathrm{AF}$ patients during the COVID-19 pandemic; drug-drug interactions involving anticoagulants, antiarrhythmics, and antivirals for COVID-19 therapy; and COVID-19 vaccination in anticoagulated $\mathrm{AF}$ patients.

\section{Search strategy and expert consensus development}

A systematic investigation of all published literature was conducted to minimize the risk of bias. A detailed search of databases (PubMed, CENTRAL, Google Scholar and UpToDate) and internet resources (websites of the American College of Cardiology [https://www.acc.org], American Heart Association [https://www.heart. org], European Society of Cardiology [https:// www.escardio.org], Centers for Disease Control and Prevention [https://www.cdc.gov], World Health Organization [https://www.covid19.who. int], European Center for Disease Prevention and Control [https://www.ecdc.eu], National Institutes of Health [https://www.covid19guidelines. nih.gov], Infectious Diseases Society of America [https://www.idsociety.org], Polish Association of Epidemiologists and Infectiologists [https://www. pteilchz.org.pl], Agency for Health Technology and Tariff System [https://www.aotm.gov.pl], Medscape [https://www.medscape.com], and browser Google [https://www.google.com]), covering the period up to $1^{\text {st }}$ May 2021 was conducted by four independent investigators (A.T.K., M.K., J.D.K., and A.K.C.). Particular attention was paid to clinical guidelines, other expert consensus documents, narrative or systematic reviews, and to studies on drug-drug interactions. The following keywords were applied: 'atrial fibrillation', 'coronavirus', 'SARS-CoV-2', 'anticoagulant', 'anticoagulation', 'NOAC', 'DOAC', 'warfarin', 'drug-drug interaction', 'antiarrhythmic', 'antiviral', and 'vaccine'. References of retrieved resources were searched manually for additional relevant publications.

Recommendations were made after extensive discussion of available scientific evidence by all authors. The manuscript was drafted by the first three listed authors (A.T.K., M.K., and J.D.K.) and the last one (A.K.C.). All authors critically revised, corrected, and accepted the manuscript. 
Table 1. Recommendations regarding anticoagulation in atrial fibrillation (AF) patients during the coronavirus disease 2019 (COVID-19) pandemic.

1. In non-infected patients, it is advisable to switch from VKAs to NOACs whenever possible due to their consistent benefits and safety with fixed dosing and no monitoring.

2. Anticoagulation principles in quarantined or asymptomatic individuals remain unchanged and NOACs are the preferred anticoagulants in the vast majority of AF patients.

3. We recommend continuing NOAC therapy in mildly symptomatic COVID-19-infected AF patients who do not require hospitalization.

4. We recommend continuing NOAC treatment or switching to therapeutic dosing of LMWH in AF patients hospitalized due to mild or moderate COVID-19 pneumonia. When deciding about anticoagulation, potential interactions of NOACs with anti-COVID-19 medications and co-morbidities (e.g., impaired renal function) should be considered.

5. We recommend anticoagulation with either LMWH or UFH in critically ill COVID-19 patients with AF hospitalized in intensive care units.

6. In AF patients treated with VKA and admitted to the hospital due to COVID-19 infection, including those with prosthetic heart valves or moderate/severe mitral stenosis, we suggest switching anticoagulation to LMWH or UFH.

7. The anticoagulation treatment strategy in COVID-19 patients with AF, particularly in critical scenarios, should be individualized based on possible drug-drug interactions.

LMWH - low-molecular-weight heparin; NOAC — non-vitamin K antagonist oral anticoagulant; UFH — unfractionated heparin; VKA - vitamin K antagonist

\section{Problems with blood test assessment during the COVID-19 pandemic in anticoagulated patients}

The current pandemic and deliberate home isolation restrict mobility and reduce access to medical care professionals (both general practitioners and hospitals), as well as services (pharmacies, laboratories, and nursing care). During the COVID-19 pandemic, a substantial number of $\mathrm{AF}$ patients treated with vitamin $\mathrm{K}$ antagonists (VKAs) did not have their international normalized ratio (INR) assessed to avoid unnecessary contact with other people. Based on 2 years of monitoring, a 12-week INR follow-up interval (using a detailed protocol with titration) extension appears feasible for a subset of patients [7]. Therefore, if it is not possible to switch to non-vitamin $\mathrm{K}$ antagonist oral anticoagulant (NOAC) treatment, we suggest measuring INR every 6-12 weeks due to logistic problems in such patients. Patients with heart failure may not be suitable for this intervention [7].

\section{Are there any interactions between} the SARS-CoV-2 virus and NOACs?

If an $\mathrm{AF}$ patient treated with NOACs is infected with SARS-CoV-2, a healthcare provider should consider both the patient's individual symptoms and medical history and then decide whether and how the therapy needs to be modified. Until such a decision is made, the previous NOAC treatment should be continued.

\section{Anticoagulation in non-COVID-19 patients and in quarantined, asymptomatic, or mildly symptomatic non-hospitalized individuals}

It is advisable to switch away from VKAs to NOACs in non-COVID-19 patients with AF whenever possible due to their consistent benefits and safety with fixed dosing and no monitoring [8]. In general, anticoagulation principles in quarantined or asymptomatic individuals remain unchanged and NOACs are the preferred anticoagulants in the vast majority of AF patients (Table 1). Moreover, we recommend continuing NOAC therapy in mildly symptomatic COVID-19-infected AF patients who do not require hospitalization.

Regular kidney function assessments are essential for the safety of NOAC therapy. It is recommended to use the Cockroft-Gault equation for estimation of creatinine clearance $(\mathrm{CrCl})$. In NOAC-treated patients without any history of kidney dysfunction, $\mathrm{CrCl}$ should be evaluated at least annually. In case of a clinically relevant decline in $\mathrm{CrCl}$, an adjustment of NOAC dose should be considered. In patients with $\mathrm{CrCl} \leq 60 \mathrm{~mL} / \mathrm{min}$, a more frequent assessment of renal function is advised. The minimum period between successive kidney function assessments in months may be calculated by dividing $\mathrm{CrCl}$ by 10 . In patients with co-existing risk factors (e.g., older age, frailty, and multiple comorbidities), more frequent monitoring is suggested [8-10]. 


\section{Optimal anticoagulation in hospitalized COVID-19 patients with AF}

We recommend continuing NOAC treatment or switching to therapeutic dosing of low-molecular-weight heparin (LMWH) in AF patients hospitalized due to mild or moderate COVID-19 pneumonia. The choice of anticoagulant should be individualized. Potential interactions of NOACs with anti-COVID-19 medications and co-morbidities (e.g., impaired renal function) should be considered. This recommendation is in line with opinions of other experts [11].

On the other hand, in severely ill patients hospitalized in intensive care units who are frequently intubated and ventilated, intravenous or subcutaneous dosing is preferable to oral, which is why the treatment of choice is either LMWH or unfractionated heparin (UFH) [12-14].

In AF patients treated with VKA and admitted to the hospital due to COVID-19 pneumonia, including those with prosthetic heart valves or moderate/severe mitral stenosis, we suggest switching anticoagulation to LMWH or UFH. This recommendation is primarily based on frequent interactions between VKAs and drugs used to treat COVID-19 infection (Central illustration).

Importantly, acute kidney injury frequently occurs in patients hospitalized due to COVID-19 infection. Its prevalence ranges from $9 \%$ to $35 \%$ of patients, depending on disease severity [15]. It should be emphasized that in patients with impaired renal function, doses of both NOACs and LMWHs should be adjusted or even therapy should be stopped, depending on the value of $\mathrm{CrCl}$. On the other hand, although intravenous therapy with UFH requires activated partial thromboplastin time monitoring, it may be safely used regardless of kidney function and - in case of bleeding - its action may be quickly reversed with protamine sulfate. Notably, specific reversal agents for NOACs have been developed, i.e., the more widely available idarucizumab for factor IIa inhibitor (dabigatran) and the less readily available andexanet alfa for the factor Xa inhibitors (rivaroxaban, apixaban, edoxaban) [16].

According to dabigatran labels, the capsules should not be opened - whereas apixaban tablets can be crushed and given via gastric tube. However, intensive care unit patients may have poor or fluctuating renal function; dabigatran is contraindicated in patients with renal function $<30 \mathrm{~mL} / \mathrm{min}$, while apixaban and rivaroxaban are contraindicated if $\mathrm{CrCl}$ is $<15 \mathrm{~mL} / \mathrm{min}$ [8].
COVID-19 patients frequently present with markedly elevated concentration of D-dimer. In this setting, D-dimer is regarded as a biomarker of disease severity and mortality $[17,18]$. Optimal management strategies in COVID-19 patients with high concentrations of $\mathrm{D}$-dimer remain unclear, particularly in those with $\mathrm{AF}$ and on therapeutic anticoagulation.

\section{Current therapy of COVID-19 pneumonia}

Treatment of COVID-19 infection has substantially evolved over time. Numerous experimental anti-COVID-19 therapies (azithromycin, chloroquine, convalescent plasma, hydroxychloroquine, interferon beta, lopinavir/ritonavir) have been demonstrated to be ineffective. Some of them had considerable potential for interactions with anticoagulants and antiarrhythmics and were used in $\mathrm{AF}$ patients.

Contemporary management of mildly symptomatic COVID-19 patients includes rehydration, antipyretic and antitussive drugs, and inhaled budesonide [19]. Primary medications recommended for moderate or severe COVID-19 pneumonia are remdesivir, tocilizumab, dexamethasone, or methylprednisolone. Additionally, the large majority of hospitalized patients require respiratory support (i.e., oxygen supplementation through a nasal cannula, face mask or venturi mask, high-flow oxygen therapy, non-invasive ventilation, mechanical ventilation, or extracorporeal membrane oxygenation). Antibiotics should be restricted to COVID-19 patients with confirmed or suspected bacterial co-infection.

\section{Are there any interactions between NOACs and antivirals or antibiotics used in COVID-19 patients?}

Both LMWHs and UFH are free of interactions with drugs used in COVID-19 therapy (Central illustration). However, they require parenteral administration and may cause heparin induced thrombocytopenia. On the other hand, multiple drug-drugs interactions of VKAs are well known. Although numerous potential interactions between NOACs and medications used for COVID-19 therapy were suggested, no detailed information has been available so far. Unfortunately, dedicated drug-drug interaction studies are lacking. Therefore, we relied on the low-quality evidence summarized on the website of The Liverpool Drug Interaction Group [20]. 


\begin{tabular}{|c|c|c|c|c|c|c|c|c|c|c|c|c|c|c|c|c|}
\hline & 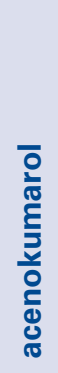 & $\begin{array}{l}\frac{1}{\pi} \\
\frac{0}{\pi} \\
\frac{x}{2} \\
\frac{x}{\pi}\end{array}$ & 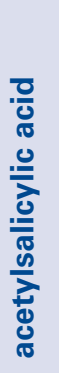 & $\begin{array}{l}\overline{0} \\
\text { 응 } \\
\text { 응 } \\
\text { 은 }\end{array}$ & $\begin{array}{l}\frac{5}{\pi} \\
\frac{\pi}{2} \\
\frac{0}{0} \\
\frac{0}{0} \\
\frac{\pi}{0}\end{array}$ & 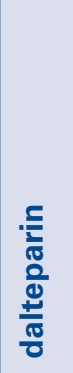 & 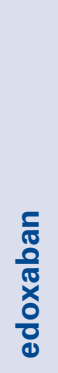 & 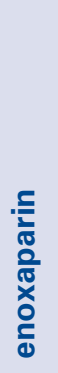 & 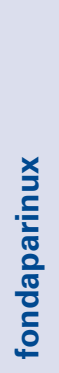 & 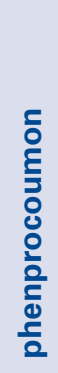 & $\begin{array}{l}\overline{0} \\
\frac{0}{5} \\
0 \\
\frac{0}{2} \\
\frac{\pi}{2}\end{array}$ & 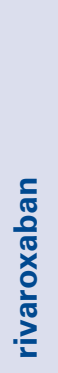 & 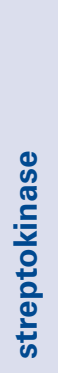 & 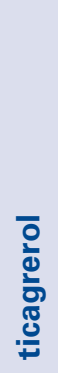 & 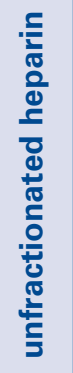 & $\begin{array}{l}\frac{5}{\frac{5}{2}} \\
\frac{\frac{1}{\frac{1}{7}}}{3} \\
\frac{\pi}{3}\end{array}$ \\
\hline \multicolumn{17}{|l|}{ acetaminophen } \\
\hline amoxicillin* & $\uparrow$ & & & & & & & & & $\uparrow$ & & & & & & $\uparrow$ \\
\hline amoxicillin/clavulanic acid* & $\uparrow$ & & & & & & & & & $\uparrow$ & & & & & & $\uparrow$ \\
\hline azithromycin* & $\uparrow$ & $\uparrow$ & & & $\uparrow$ & & $\uparrow$ & & & $\uparrow$ & & $\uparrow$ & & & & $\uparrow$ \\
\hline cefuroxime* & $\uparrow$ & & & & & & & & & $\uparrow$ & & & & & & $\uparrow$ \\
\hline ceftriaxone* & $\uparrow$ & & & & & & & & & $\uparrow$ & & & & & & $\uparrow$ \\
\hline dexamethasone & $\uparrow$ & $\downarrow$ & $\uparrow$ & & $\downarrow$ & & $\downarrow$ & & & $\uparrow$ & & $\downarrow$ & & & & $\uparrow$ \\
\hline levofloxacin* & $\uparrow$ & & & & & & & & & $\uparrow$ & & & & & & $\uparrow$ \\
\hline meropenem* ${ }^{*}$ & $\uparrow$ & & & & & & & & & $\uparrow$ & & & & & & $\uparrow$ \\
\hline methylprednisolone & $\uparrow$ & $\downarrow$ & $\uparrow$ & & $\downarrow$ & & $\downarrow$ & & & $\uparrow$ & & $\downarrow$ & & & & $\uparrow$ \\
\hline piperacillin/tazobactam* & $\uparrow$ & & & & & & & & & $\uparrow$ & & & & & & $\uparrow$ \\
\hline \multicolumn{17}{|l|}{ remdesivir } \\
\hline tocilizumab & $\downarrow$ & $\downarrow$ & & $\downarrow$ & & & & & & $\downarrow$ & $\downarrow$ & $\downarrow$ & & $\downarrow$ & & $\downarrow$ \\
\hline
\end{tabular}

Central illustration. Interactions between anticoagulants, antiplatelets, fibrinolytics and drugs used to treat coronavirus disease 2019 (COVID-19) patients. Notably, the overall quality of this evidence is very low. This table is based on the COVID-19 Drug Interaction website of the University of Liverpool (www.covid19-druginteractions.org). Gray color: No information found on the website, but interaction seems unlikely according to the summary of product characteristics. Green color: no clinically significant interaction is expected, or potential interaction is likely to be of weak intensity, not requiring additional action/monitoring or dose adjustment. Yellow color: Potential weak interaction which may require additional monitoring (e.g., more frequent international normalized ratio monitoring if on vitamin $\mathrm{K}$ antagonist). Orange color: Potential interaction which may require dose adjustment. Red color: The drugs should not be co-administered. $\uparrow$ Potential increased exposure to the anticoagulant, antiplatelet, or fibrinolytic drugs. $\downarrow$ Potential decreased exposure to the anticoagulant, antiplatelet, or fibrinolytic drugs. *Antibiotics are only indicated in COVID-19 patients if bacterial co-infection is present.

P-glycoprotein (P-gp) inhibitors are likely to increase dabigatran concentration and their use may expose a patient treated with a standard dabigatran dose to the elevated risk of bleeding. Therefore, in such patients we recommend careful clinical surveillance (aimed at early detection of signs and symptoms of bleeding) and/or consideration of dabigatran dose reduction. On the other hand, P-gp inducers may reduce dabigatran blood concentration. As a consequence, potent P-gp inducers should not be co-administered with dabigatran.

Importantly, dabigatran is unlikely to exert any drug-drug interaction related to the cytochrome P450.

No relevant literary citations were identified in either MEDLINE or in EMBASE with keywords indicating NOACs and antiviral agents used for COVID-19 therapy.

No relevant literary citations were identified in MEDLINE and EMBASE with terms indicating NOACs and antivirals. Particularly, remdesivir has no clinically significant drug interactions documented, likely due to its rapid clearance.

\section{Are there any interactions between NOACs and tocilizumab?}

The tocilizumab's summary of product characteristics indicates that tocilizumab interacts with cytochrome P450, but any information on drug-drug interaction involving P-gp transport is lacking [21]. Importantly, neither NOACs nor tocilizumab 
manufacturers have conducted dedicated studies on interactions between the drugs. The dabigatran's label seems to indicate that there is no interaction of dabigatran related to the cytochrome P450. According to tocilizumab's summary of product characteristics, tocilizumab interacts with cytochrome P450; no information on drug-drug interaction related to P-gp transport was available. According to dabigatran's label, dabigatran is not expected to have any cytochrome P450 related drug-drug interactions. Tocilizumab may increase warfarin metabolism.

\section{Interactions between azithromycin and antiarrhythmic drugs}

Although the initial enthusiasm towards azithromycin as an experimental COVID-19 therapy has been tempered by neutral results in randomized clinical trials [22-24], its use may still be considered in patients with concomitant bacterial co-infection. Prolonged cardiac repolarization and prolonged QT intervals pose a risk of developing cardiac arrhythmia and torsades de pointes. Azithromycin — as with other macrolides - may lead to an increased risk of ventricular arrhythmias, including torsade de pointes, and hence to cardiac arrest. Azithromycin is not contraindicated but should be used with caution in patients with ongoing proarrhythmic conditions - especially elderly patients and women: (1) receiving treatment with other drugs known to prolong QT intervals, such as antiarrhythmics of class IA (quinidine and procainamide) and class III (dofetilide, amiodarone, and sotalol); (2) with electrolyte disturbances, particularly in patients with hypokalemia or hypomagnesemia; (3) with significant bradycardia, cardiac arrhythmia or severe heart failure. Several observational studies have shown a rare short-term risk of arrhythmia, myocardial infarction, and mortality of cardiovascular causes associated with azithromycin. These findings should be taken into account and balanced against the clinical benefits when administering azithromycin.

\section{Antiarrhythmic drugs used in the rhythm control strategy}

An integral part of AF treatment is restoring sinus rhythm. Rhythm control therapy is often necessary to improve symptoms in AF patients who are symptomatic on rate control therapy. Importantly, the underlying hypoxemia, inflam- mation, and dyselectrolytemia (i.e., hypokalemia, hypomagnesemia, and acidosis) may be triggers for $\mathrm{AF}$ in patients with COVID-19 infection [11]. They should be corrected if possible and then if AF persists, therapy with antiarrhythmics may be considered. In the setting of COVID-19 infection, chemical cardioversion is preferred over its electrical counterpart as the first choice AF management in hemodynamically stable patients [31]. Amiodarone is a known substrate for both CYP3A4 and CYP2C8. The concomitant use of drugs inhibiting these isoenzymes may inhibit amiodarone metabolism and increase its concentration and exposure [25-28]. The document of product characteristics recommends to avoid or to pay extreme caution with the concomitant use of amiodarone with CYP3A4 inhibitors. Interactions between amiodarone and inhibitors of CYP3A4 may be observed for several months after discontinuation of such treatment due to the long half-life of amiodarone.

Propafenone is known to be metabolized by CYP2D6, CYP3A4, and CYP1A2 enzymes. The concomitant use of drugs inhibiting these isoenzymes leads to increased plasma levels of propafenone [28, 29]. Patients should be carefully monitored and the dose adjusted accordingly when propafenone is used with inhibitors of these enzymes. The summary of product characteristics warns against the simultaneous use of propafenone with CYP2D6 and CYP3A4 inhibitors. Moreover, co-administration of CYP3A4 inhibitors may increase exposure to propafenone and the risk of QRS complex prolongation on electrocardiography, dangerous complex ventricular arrhythmias, and blockage of beta-blockers. Adequate caution is necessary if these drugs are used together. Low-doses of propafenone may be considered in selected patients diagnosed with COVID-19 if the electrocardiography is carefully monitored for the early recognition of acquired QTc prolongation, torsade de pointes, and other dangerous ventricular arrhythmias.

In summary, antiarrhythmics have mild interactions with tocilizumab, moderate interactions with steroids, and moderate or severe interactions with antibiotics (Fig. 1). On the other hand, no interactions between antiarrhythmics and remdesivir were reported.

\section{Antiarrhythmic drugs used in the rate control strategy}

Both verapamil and diltiazem have been shown to be inhibitors of CYP3A4 enzymes and P-gp. 


\begin{tabular}{|c|c|c|c|c|c|}
\hline & amiodaron & digoxin & lidocaine & propafenone & quinidine \\
\hline \multicolumn{6}{|l|}{ acetaminophen } \\
\hline amoxicillin* & & $\uparrow$ & & & \\
\hline amoxicillin/clavulanic acid* & & $\uparrow$ & & & \\
\hline azithromycin* & ! & $\uparrow$ & & & ! \\
\hline \multicolumn{6}{|l|}{ cefuroxime* } \\
\hline \multicolumn{6}{|l|}{ ceftriaxone* } \\
\hline dexamethasone & $\downarrow \#$ & $\hat{\imath}$ & $\#$ & \# & $\#$ \\
\hline levofloxacin* & $!$ & $\uparrow$ & & & \\
\hline meropenem* & & $\uparrow$ & & & \\
\hline methylprednisolone & \# & $\hat{\imath}$ & $\#$ & \# & \# \\
\hline \multicolumn{6}{|l|}{ piperacillin/tazobactam* } \\
\hline \multicolumn{6}{|l|}{ remdesivir } \\
\hline tocilizumab & $\downarrow$ & & $\downarrow$ & & \\
\hline
\end{tabular}

Figure 1. Interactions between antiarrhythmics and drugs used to treat coronavirus disease 2019 (COVID-19) patients. Notably, the overall quality of this evidence is very low. This table is based on the COVID-19 Drug Interaction website of the University of Liverpool (www.covid19-druginteractions.org). Gray color: No information was found on the website, but interaction seems unlikely according to the summary of product characteristics. Green color: no clinically significant interaction is expected, or potential interaction is likely to be of weak intensity, not requiring additional action/monitoring or dose adjustment. Yellow color: Potential weak interaction which may require additional monitoring. Orange color: Potential interaction which may require dose adjustment. Red color: The drugs should not be co-administered. $\uparrow$ Potential increased exposure to the antiarrhythmic drug. $\downarrow$ Potential decreased exposure to the antiarrhythmic drug. ${ }^{*}$ Antibiotics are only indicated in COVID-19 patients if bacterial co-infection is present. ! Both drugs (when co-administered) may lead to marked QT interval prolongation and increase the risk of torsades de pointes. \#Steroids may cause hypokalemia, which increases the risk of torsades de pointes with antiarrhythmics. ^ Steroids may cause hypokalemia, which increases the risk of digoxin toxicity.

Simultaneous use of CYP3A4 inhibitors leads to elevations of their plasma levels. Doses of verapamil and diltiazem should potentially be decreased. Beta-blockers such as metoprolol, carvedilol, and propranolol are substrates for the cytochrome CYP2D6 enzyme [30]. Metoprolol is highly dependent on the CYP2D6 enzyme, with about $70-80 \%$ of its metabolism being through this pathway. The other beta-blockers are much less reliant on CYP2D6 than metoprolol. The metabolism of digoxin is not dependent on cytochrome $\mathrm{P} 450$; its elimination is mainly through the kidneys and involves P-gp [31]. It is also advisable to reduce the dose of digoxin and to continue monitoring. No dosage reduction is indicated in the case of concomitant administration of tocilizumab used in patients with COVID-19 [28].

Based on previous clinical experience and the risk of drug-drug interactions, beta-blockers are recommended as a first-line therapy for rate control strategy [32].

\section{COVID-19 vaccination in patients receiving anticoagulants}

Public Health England's Immunization Against Infectious Disease states that the vaccine may be given intramuscular to patients treated with warfarin whose latest INR is lower than the upper limit of the therapeutic range [32].

For the vaccination a thin needle should be used and then firm pressure applied to the site of injection for at least $2 \mathrm{~min}$. The information of possible hematoma from the injection should be conveyed to the patient. If the level of anticoagulation is unknown, the general practitioner or doctor in charge responsible for anticoagulant treatment should be contacted.

COVID-19 vaccine may be also given to patients who are treated with NOACs. The 2021 Update of European Heart Rhythm Association Consensus on NOACs recommends: (1) to skip the morning dose of the NOAC before intramuscular 
injection, (2) in NOACs taken twice daily, to take the morning dose $3 \mathrm{~h}$ after the vaccination especially in high risk of ischemic stroke, and (3) in NOACs taken twice a day, start NOAC with the next regular dose [8]. The rules concerning reduction the risks of hematoma after vaccination are the same as described for warfarin above.

\section{Summary}

Patients with AF due to comorbidities and elderly age are assumed to be at a higher risk of a severe course of COVID-19. In general, anticoagulation principles in quarantined or asymptomatic individuals remain unchanged. It is advisable to switch from VKAs to NOACs whenever possible due to their consistent benefits and safety with fixed dosing and no monitoring. Additionally, in AF patients hospitalized due to mild or moderate COVID-19 pneumonia, we recommend continuing NOAC treatment or switching to LMWH. On the other hand, in severely ill patients hospitalized in intensive care units, intravenous or subcutaneous dosing is preferable to oral, which is why the treatment of choice is either LMWH or UFH. Finally, particularly in critical scenarios, the treatment strategy in COVID-19 patients with AF should be individualized based on possible interactions between anticoagulants, antiarrhythmics, antivirals, and antibiotics. COVID-19 vaccination may be safely performed in anticoagulated AF patients.

\section{Conflict of interest: None declared}

\section{References}

1. Richardson S, Hirsch J, Narasimhan M, et al. Presenting characteristics, comorbidities, and outcomes among 5700 patients hospitalized with COVID-19 in the new york city area. JAMA. 2020; 323(20): 2052-2059, doi: 10.1001/jama.2020.6775.

2. Li J, He X, Zhang W, et al. Meta-analysis investigating the relationship between clinical features, outcomes, and severity of severe acute respiratory syndrome coronavirus 2 (SARS-CoV-2) pneumonia. Am J Infect Control. 2021; 49(1): 82-89, doi: 10.1016/j.ajic.2020.06.008, indexed in Pubmed: 32540370.

3. Ambrus DB, Benjamin EJ, Bajwa EK, et al. Risk factors and outcomes associated with new-onset atrial fibrillation during acute respiratory distress syndrome. J Crit Care. 2015; 30(5): 994-997, doi: 10.1016/j.jcrc.2015.06.003, indexed in Pubmed: 26138630.

4. Inciardi R, Adamo M, Lupi L, et al. Characteristics and outcomes of patients hospitalized for COVID-19 and cardiac disease in Northern Italy. Eur Heart J. 2020; 41(19): 1821-1829, doi: 10.1093/eurheartj/ehaa388.

5. Gopinathannair R, Merchant FM, Lakkireddy DR, et al. COVID-19 and cardiac arrhythmias: a global perspective on arrhythmia characteristics and management strategies. J Interv Card
Electrophysiol. 2020; 59(2): 329-336, doi: 10.1007/s10840-02000789-9, indexed in Pubmed: 32494896.

6. Onder G, Rezza G, Brusaferro S. Case-fatality rate and characteristics of patients dying in relation to COVID-19 in italy. JAMA. 2020; 323(18): 1775-1776, doi: 10.1001/jama.2020.4683, indexed in Pubmed: 32203977.

7. Porter AL, Margolis AR, Staresinic CE, et al. Feasibility and safety of a 12-week INR follow-up protocol over 2 years in an anticoagulation clinic: a single-arm prospective cohort study. J Thromb Thrombolysis. 2019; 47(2): 200-208, doi: 10.1007/ s11239-018-1760-9, indexed in Pubmed: 30368762.

8. Steffel J, Collins R, Antz M, et al. 2021 european heart rhythm association practical guide on the use of non-vitamin $\mathrm{K}$ antagonist oral anticoagulants in patients with atrial fibrillation. EP Europace. 2021, doi: 10.1093/europace/euab065.

9. Tomaszuk-Kazberuk A, Kołtowski L, Balsam P, et al. Use of nonvitamin $\mathrm{K}$ antagonist oral anticoagulants (NOACs) in patients with atrial fibrillation: Messages from the 2018 EHRA. Cardiol J. 2018; 25(4): 423-440, doi: 10.5603/CJ.2018.0080, indexed in Pubmed: 30211927.

10. Hermans C, Lambert C. Impact of the COVID-19 pandemic on therapeutic choices in thrombosis-hemostasis. J Thromb Haemost. 2020; 18(7): 1794-1795, doi: 10.1111/jth.14845, indexed in Pubmed: 32294321.

11. Gawałko M, Kapłon-Cieślicka A, Hohl M, et al. COVID-19 associated atrial fibrillation: Incidence, putative mechanisms and potential clinical implications. Int J Cardiol Heart Vasc. 2020; 100631, doi: 10.1016/j.ijcha.2020.100631, indexed in Pubmed: 32904969.

12. Tang N, Li D, Wang X, et al. Abnormal coagulation parameters are associated with poor prognosis in patients with novel coronavirus pneumonia. J Thromb Haemost. 2020; 18(4): 844-847, doi: 10.1111/jth.14768, indexed in Pubmed: 32073213.

13. Tang N, Bai H, Chen X, et al. Anticoagulant treatment is associated with decreased mortality in severe coronavirus disease 2019 patients with coagulopathy. J Thromb Haemos. 2020; 18(5): 1094-1099, doi: 10.1111/jth.14817.

14. Papakonstantinou PE, Borovac JA, Gąsecka A, et al. Anticoagulation therapy in non-valvular atrial fibrillation in the COVID-19 era: is it time to reconsider our therapeutic strategy? Eur J Prev Cardiol. 2021 [Epub ahead of print], doi: 10.1093/eurjpc/zwab021, indexed in Pubmed: 33564838.

15. Fabrizi F, Alfieri CM, Cerutti R, et al. COVID-19 and acute kidney injury: a systematic review and meta-analysis. Pathogens. 2020; 9(12), doi: 10.3390/pathogens9121052, indexed in Pubmed: 33334023.

16. Wojtowicz D, Tomaszuk-Kazberuk A, Małyszko J, et al. Hematuria and other kinds of bleedings on non-vitamin $\mathrm{K}$ antagonist oral anticoagulants in patients with atrial fibrillation: an updated overview on occurrence, pathomechanisms and management. Wiad Lek. 2020; 73(11): 2528-2534, doi: 10.36740/wlek202011135.

17. Ozen M, Yilmaz A, Cakmak V, et al. D-Dimer as a potential biomarker for disease severity in COVID-19. Am J Emerg Med. 2021; 40: 55-59, doi: 10.1016/j.ajem.2020.12.023, indexed in Pubmed: 33348224.

18. Yao Y, Cao J, Wang Q, et al. D-dimer as a biomarker for disease severity and mortality in COVID-19 patients: a case control study. J Intensive Care. 2020; 8: 49, doi: 10.1186/s40560-02000466-z, indexed in Pubmed: 32665858.

19. Flisiak R, Horban A, Jaroszewicz J, et al. Management of SARSCoV- infection: recommendations of the Polish Association of 
Epidemiologists and Infectiologists as of April 26, 2021. Pol Arch Intern Med. 2021; 131(5): 487-496, doi: 10.20452/pamw.15979, indexed in Pubmed: 33908727.

20. The Liverpool Drug Interaction Group (University of Liverpool, U., University Hospital of Basel (Switzerland) and Radboud UMC (Netherlands). [Internet]. Interactions with Experimental COVID-19 Therapies, 2021. http://www.covid19-druginteractions.org (cited 2021 May 1).

21. RECOVERY Collaborative Group. Tocilizumab in patients admitted to hospital with COVID-19 (RECOVERY): a randomised, controlled, open-label, platform trial. Lancet. 2021; 397(10285): 1637-1645, doi: 10.1016/S0140-6736(21)00676-0, indexed in Pubmed: 33933206.

22. PRINCIPLE Trial Collaborative Group. Azithromycin for community treatment of suspected COVID-19 in people at increased risk of an adverse clinical course in the UK (PRINCIPLE): a randomised, controlled, open-label, adaptive platform trial. Lancet. 2021; 397(10279): 1063-1074, doi: 10.1016/S01406736(21)00461-X, indexed in Pubmed: 33676597.

23. O'Connor S. RECOVERY Collaborative Group. Azithromycin in patients admitted to hospital with COVID-19 (RECOVERY): a randomised, controlled, open-label, platform trial. Lancet. 2021; 397(10274): 605-612, doi: 10.1016/S0140-6736(21)00149-5, indexed in Pubmed: 33545096.

24. Furtado R, Berwanger O, Fonseca $\mathrm{H}$, et al. Azithromycin in addition to standard of care versus standard of care alone in the treatment of patients admitted to the hospital with severe COVID-19 in Brazil (COALITION II): a randomised clinical trial. Lancet. 2020; 396(10256): 959-967, doi: 10.1016/s01406736(20)31862-6.
25. Hu YF, Cheng WH, Hung Y, et al. Management of atrial fibrillation in COVID-19 pandemic. Circ J. 2020; 84(10): 1679-1685, doi: 10.1253/circj.CJ-20-0566, indexed in Pubmed: 32908073.

26. Gillis AM, Kates RE. Clinical pharmacokinetics of the newer antiarrhythmic agents. Clin Pharmacokinet. 1984; 9(5): 375-403, doi: 10.2165/00003088-198409050-00001, indexed in Pubmed: 6437721.

27. Latini R, Tognoni G, Kates RE. Clinical pharmacokinetics of amiodarone. Clin Pharmacokinet. 1984; 9(2): 136-156, doi: 10.2165/00003088-198409020-00002, indexed in Pubmed: 6370540 .

28. Russo V, Rago A, Carbone A, et al. Atrial Fibrillation in COVID-19: From Epidemiological Association to Pharmacological Implications. J Cardiovasc Pharmacol. 2020; 76(2): 138-145, doi: 10.1097/FJC.0000000000000854, indexed in Pubmed: 32453074.

29. Naccarelli G, Wolbrette D, Khan M, et al. Old and new antiarrhythmic drugs for converting and maintaining sinus rhythm in atrial fibrillation: comparative efficacy and results of trials. Am J Cardiol. 2003; 91(6): 15-26, doi: 10.1016/s0002-9149(02)03375-1.

30. Shin J, Johnson JA. Pharmacogenetics of beta-blockers. Pharmacotherapy. 2007; 27(6): 874-887.

31. Iisalo E. Clinical pharmacokinetics of digoxin. Clin Pharmacokinet. 1977; 2(1): 1-16, doi: 10.2165/00003088-197702010-00001, indexed in Pubmed: 322907.

32. Rattanawong P, Shen W, El Masry H, et al. Guidance on shortterm management of atrial fibrillation in coronavirus disease 2019. J Am Heart Assoc. 2020; 9(14): e017529, doi: 10.1161/ JAHA.120.017529, indexed in Pubmed: 32515253. 